\title{
Mujeres que cuidaban a víctimas de violencia, mujeres acosadas en programas psicosociales chilenos
}

\author{
José Matamala Pizarro ${ }^{1}$, Alba Barrera Lagos² \\ Pontificia Universidad Católica de Valparaíso- Chile
}

Se exponen resultados de una investigación cualitativa cuyo objetivo fue analizar y comprender la manifestación del acoso laboral en mujeres de programas psicosociales chilenos que brindaban trabajo de cuidado a víctimas de violencia. El muestreo se realizó de manera intencionada y por conveniencia. Se realizaron entrevistas semiestructuradas a 10 mujeres de programas psicosociales del SENAME y SERNAMEG. Se realizó análisis de contenidotemático con los datos construidos. Los resultados mostraron que la exposición al acoso laboral fue estigmatizante, perpetradas principalmente por hostigamiento al trabajo, afrontadas preferentemente con estrategias defensivas individuales. Las condiciones laborales fueron sindicadas como las principales causantes del acoso laboral. Se concluye que involucró perjuicios para la salud mental y los derechos de las trabajadoras.

Palabras claves: violencia laboral, acoso laboral, sufrimiento en el trabajo, programas psicosociales, salud mental en el trabajo.

\section{Women who to care victims of violence, women harassed at chilean psychosocial programs}

The results of a qualitative research were exposed, whose objective was to analyze and understand the manifestation of workplace harassment in women of Chilean psychosocial programs that provided care work to victims of violence. Sampling was done intentionally and for convenience. Semi-structured interviews were conducted with 10 women from psychosocial programs of SENAME and SERNAMEG. The content-thematic analysis was carried out for the constructed data. The results showed that the exposure to workplace harassment was stigmatizing, mainly perpetrated by harassment to work, faced preferably with individual defensive strategies. The working conditions were syndicated as the main causes of workplace harassment. It is concluded that it involved damage to mental health and the rights of the workers.

1 Magíster en Psicología, mención comunitaria, Universidad de Chile. Doctorando en Psicología, Pontificia Universidad Católica de Valparaíso, Chile. Docente adjunto en Escuela de Psicología de la Universidad Católica Silva Henríquez y Universidad de Santiago de Chile. Dirección postal: 2340000. Contacto: jose.matamala@pucv.cl https://orcid. org/0000-0003-1369-4219

2 Trabajadora Social. Doctoranda en Psicología, Pontificia Universidad Católica de Valparaíso, Chile. Diplomada en metodologías para la Investigación en ciencias sociales, Pontificia Universidad Católica de Valparaíso. Docente adjunta en Escuela de Trabajo Social, Universidad de las Américas, Viña del Mar, Chile. Dirección postal: 2340000. Contacto: alba.barrera.l@ mail.pucv.cl https://orcid.org/0000-0003-1993-1406 
Keywords: workplace violence, workplace harrasment, suffering at work, psychosocial programs, mental health at work.

Mulheres que cuidavam de vítimas de violência, mulheres assediadas em programas psicossociais chilenos

Os resultados de uma pesquisa qualitativa foram expostos, cujo objetivo foi analisar e compreender a manifestação de assédio no trabalho em mulheres de programas psicossociais chilenos que prestavam assistência a vítimas de violência. A amostragem foi feita intencionalmente e por conveniência. Entrevistas semi-estruturadas foram realizadas com 10 mulheres de programas psicossociais de SENAME e SERNAMEG. A análise temática de conteúdo foi realizada com os dados construídos. Os resultados mostraram que a exposição ao assédio no trabalho foi estigmatizante, principalmente perpetrada pelo assédio ao trabalho, enfrentado preferencialmente com estratégias defensivas individuais. As condiçóes de trabalho foram sindicadas como as principais causas de assédio no trabalho. Conclui-se que envolveu danos à saúde mental e aos direitos dos trabalhadores.

Palavras-chave: violência no trabalho, assédio moral no trabalho, sofrimento no trabalho, programas psicossociais, saúde mental no trabalho.

Femmes qui se sont occupées de victimes de violence, femmes harcelées dans les programmes psychosociaux chiliens

Les résultats d'une recherche qualitative ont été exposés, dont l'objectif était d'analyser et de comprendre la manifestation du harcèlement sur le lieu de travail chez les femmes de programmes psychosociaux chiliens qui fournissaient des soins aux victimes de violence. L'échantillonnage a été fait intentionnellement et par commodité. Des entretiens semistructurés ont été menés avec 10 femmes des programmes psychosociaux du SENAME et de SERNAMEG. L'analyse thématique du contenu a été réalisée pour les données construites. Les résultats ont montré que l'exposition au harcèlement sur le lieu de travail était stigmatisante, principalement perpétrée par le harcèlement au travail, confrontée de préférence à des stratégies de défense individuelles. Les conditions de travail étaient la principale cause de harcèlement au travail. Il est conclu qu'il s'agissait de dommages à la santé mentale et aux droits des travailleurs.

Mots-clés: violence au travail, harcèlement au travail, souffrance au travail, programmes psychosociaux, santé mentale au travail 
El acoso moral en el trabajo (en adelante AMT) es una forma de violencia en el trabajo (Chappell y Di Martino, 2006) que involucra una transgresión en la integridad anímica y los derechos de los trabajadores, quienes al ser victimizados por AMT sienten que se les maltrató, despreció, humilló o rechazó (Hirigoyen, 2013). La Organización Internacional del Trabajo (OIT) señala que el AMT al basarse en relaciones de poder, normas culturales y estereotipos de género y desigualdades, se acrecienta mundialmente como factor de riesgo psicosocial para la salud en el trabajo (OIT, 2018).

En la comprensión del AMT, un autor relevante ha sido Heinz Leymann (1990). Leymann comentó que el acoso laboral o mobbing se presenta en los contextos laborales para atacar de forma prolongada y repetitiva en el tiempo a un trabajador con el objetivo de infundirle terror psíquico. Leymann (1996) sostuvo que el mobbing se presenta en un curso estereotípico organizado en fases: 1) Incidentes críticos: eventos hostiles que permiten allanar el terreno para la escalada y mantención de la violencia que representa el acoso laboral. 2) Estigmatización y acoso: los comportamientos no siempre son identificados como acoso gracias al uso de la manipulación agresiva. Estos actos se promueven durante un tiempo, lo que causa la estigmatización en la víctima. 3) Intervención de la administración de personal: las direcciones de la organización intervienen. Sin embargo, es habitual que terminen por responsabilizar a la víctima de lo ocurrido. Cuando no se logra un cambio, las víctimas solicitan ayuda especializada a profesionales médicos, abogados u otros. 4) Salida de la organización o expulsión: corresponde a la salida de la persona acosada de la empresa.

Otra autora importante en la delimitación del acoso laboral ha sido Marie-France Hirigoyen. Hirigoyen (2013) menciona que es preferible denominar al acoso laboral como acoso moral en el trabajo (AMT), puntualizando en que es una forma de violencia que no se debe tolerar 
ni validar. Para ella, el AMT puede llegar a encubrir un conflicto que está soterrado sin resolución. Hirigoyen (2013) también agrega que el AMT se caracteriza por la responsabilidad en la perpetración: en el AMT vertical ascendente, las conductas de acoso se dirigen hacia un jefe; en el AMT vertical descendente, los superiores son los hostigadores; en el AMT horizontal, los pares se acosan y por último está el AMT mixto. Cabe consignar que estas situaciones no forman parte de acciones de descalificación aisladas o eventuales, puesto que buscan causar un daño severo a la integridad moral del individuo (Albanaes et al.,2017), por lo que son conductas de hostigamiento recurrentes.

Tal como lo indica Hirigoyen (2016), las acciones hostiles en el AMT son desarrolladas por personas que en general no tienen problemas psicopatológicos ni son necesariamente malvadas. Aquellas realizan un trabajo sucio (Dejours, 2009b) distinguido por acciones inmorales con la finalidad de mantener su puesto de trabajo; controlar los tiempos de producción o acceder a beneficios. En algunos casos, los perpetradores pueden reconocer que recurren a la rudeza y el autoritarismo sin querer causar daños, sino para que se interioricen las normas y mandatos de la institución (Fernández et al., 2012).

Los perpetradores ejecutan las conductas de hostigamiento de cuatro formas (Hirigoyen, 2016): 1) El aislamiento e impedimento de la comunicación de la víctima. 2) Realización de humillaciones, degradación y descalificación para dańar sus características personales. 3) Usar el hostigamiento para atacar las condiciones de trabajo. 4) Intimidar para aterrorizar al resto de colegas.

Estas cuatro maneras son agrupadas en dos grandes tácticas de hostigamiento: por ataque personal y por ataque al trabajo (Branch, Ramsay y Barker, 2013). La primera involucra los dos primeros componentes, mientras los dos últimos conforman la segunda táctica.

Siguiendo a Hirigoyen (2013), las víctimas suelen ser mujeres muy comprometidas con su trabajo. Con el AMT se las fuerza a trabajar mal, a realizar acciones que entran en contradicción con sus principios, lo que aminora sus posibilidades de reconocimiento y aumenta su 
sufrimiento (Dejours, 2006). Por esa razón, es que las víctimas de AMT recurren a diferentes estrategias para su afrontamiento.

El sufrimiento es soportable solo si se logra conquistar una normalidad (Dejours, 2009a). La conservación de esta normalidad sufriente se logra con la utilización de estrategias defensivas (Dejours y Gernet, 2012). En el caso de los mecanismos defensivos para soportar el AMT (Hirigoyen, 2016) destacan estrategias individuales y colectivas (Dejours, 2014; Molinier, 2010), tales como la negación del sufrimiento colectivo, el hiperactivismo o hipoactivismo, la virilidad o muliebridad defensiva, la inhibición de acciones colectivas.

En otro ámbito, algunos factores organizacionales que facilitan el AMT tienen relación con el liderazgo y la cultura organizacional. El AMT se desarrolla preferentemente en organizaciones que favorecen el liderazgo destructivo o tóxico (Krasikova, Green y LeBreton, 2013) y la supervisión abusiva (Tepper, Simon, y Park, 2017). La gestión organizacional o management basada en la eficiencia, competencia empresarial, la precarización laboral, el servicio de atención de público y pacientes, la rapidez en la producción y resolución de problemas constituyen factores de riesgos relacionados con la violencia en el trabajo (Toro y Gómez, 2016). Otros factores involucrados en el AMT tienen relación con organizaciones que favorecen la violencia de género (dos Santos y Ducatti, 2014; Díaz et al., 2017) o que sancionan la participación en organizaciones sindicales y/o el goce de derechos, como el descanso por maternidad (López y Seco, 2016).

Las consecuencias del AMT se observa en aparición de patologías en salud mental como estrés, alteraciones del ciclo de sueño-vigilia, depresión, fatiga y desgaste mental o burnout, disminución del bienestar (Appelbaum et al., 2012; Appelbaum et al., 2012b). El AMT también implica afecciones en los ámbitos familiares (Silva et al., 2017) y sociales (Madrigal y Elizondo, 2015). Aquello incide en aumentos en las tasas de absentismo laboral, en las cifras de denuncias efectuadas en tribunales laborales, en presentación de licencias médicas y gastos en salud pública (OIT, 2016). 
Algunas investigaciones han señalado que el AMT abarca consecuencias diferenciadas según el género, siendo usual que se relacione con la violencia de género hacia las mujeres (Molina y Jung, 2015; dos Santos y Ducatti, 2014; Hernandez, 2014). Otros estudios han reportado que el AMT prevalece en la organización del trabajo de servicios (Ros, 2013; Eurofound, 2015) y en particular en aquellos trabajos de cuidados de personas, tales como las labores de enfermería (Dois, 2012), de medicina (Sepúlveda et al., 2017) y técnica paramédica (Campo y Klijn, 2018).

El tipo de trabajo de cuidado (Molinier y Legarreta, 2016) al que se refiere este estudio, es realizado principalmente por mujeres profesionales de la psicología y el trabajo social en la atención de víctimas de violencia en programas psicosociales tercerizados por el Estado chileno. Los cuidados que ejecutan en los programas vinculados con la política pública de infancia tienen relación con procesos psicoterapéuticos y psicosociales con niños y niñas vulnerados en sus derechos, quienes fueron objeto de maltrato grave, negligencias, exclusión escolar, violencia sexual, entre otras afecciones. En los programas relacionados con la política pública de mujer y equidad de género, efectúan procedimientos similares con la diferencia que en estos centros se atienden mujeres que han sido víctimas de violencia de género propiciadas principalmente por sus parejas.

Cabe señalar que la mayoría de los programas psicosociales son licitados en concursos públicos, instancias en las que participan diferentes organismos privados — como ONG, fundaciones, corporaciones, etc.los que terminan ejecutando la política pública. En ese sentido, el trabajo de cuidado se realiza de manera externalizada, quedando como una responsabilidad privada el desarrollo de una política de interés social. A esta forma de administración de la política pública se le conoce como New Public Management. Fardella et al., (2016) señalan que esta forma de administración implica que los proveedores entre en una alta competencia, orientándose a resultados medidos a través de instrumentos de evaluación y de rendición de cuentas, donde el Estado se convierte en un cliente que decide qué se administra en función del sistema de medición 
o de rendición de cuentas (accountability). Por esa razón, en el área de los programas psicosociales, el gobierno abre las licitaciones para que los organismos privados compitan y accedan a financiamiento. Este solo se mantiene mientras dura el contrato que establece el organismo privado con el servicio y realicen los intereses del Estado, lo que es supervisado a través de registros de actividad y cumplimiento de metas, inspecciones de gastos y coberturas (Fardella et al., 2016).

Por esa razón, la literatura científica ha señalado los riesgos que se acrecientan en estos dispositivos, dado que el trabajo que allí se realiza tensiona a los y las trabajadoras. El trabajo de cuidado en programas psicosociales ha sido destacado por la alta carga emocional por atender población victimizada (Quintana, 2005; Santana y Farkas, 2007); con la preocupación por los resultados, la rendición de cuentas y las metas que reducen las identidades laborales a substancias calculables (Fardella et al., 2016), el síndrome de burnout (Bilbao et al., 2018), la flexibilidad laboral de profesionales jóvenes (Sisto y Fardella, 2008), como la precariedad laboral (Pavez, 2018), la que implica que los y las trabajadoras no tengan un vínculo laboral directo con el Estado, sino con organismos privados que no logran asegurar las condiciones laborales más proclives para el correcto desarrollo de su trabajo.

Pese a lo enunciado, los estudios nacionales realizados en este ámbito laboral no han profundizado en el AMT, pese a que en un trabajo anterior se indicó que la problemática está presente en el rubro. En esa brecha, es que el presente artículo expone resultados de una investigación cuyo objetivo fue analizar y comprender la manifestación del AMT en mujeres que trabajaban en programas psicosociales del SENAME y del Servicio Nacional de la Mujer SERNAMEG cuya misión era brindar cuidado a víctimas de violencia. Este trabajo forma parte del proyecto de investigación "La paradoja de la violencia: acoso moral en el trabajo en profesionales que intervienen violencia" desarrollado en el marco de los estudios doctorales de ambos investigadores. Con la presentación de los resultados, se busca aportar evidencia que permita a la institucionalidad nacional y los colectivos de trabajadores/ as abordar esta problemática. 


\section{Método}

El estudio está basado en el enfoque de investigación cualitativa (Bassi, 2014), puesto que se buscó comprender cómo las participantes experimentaron ciertos eventos y condiciones — la manifestación del AMT - y cómo la manejaron y afrontaron. El diseño de investigación privilegió el estudio de los relatos de las participantes. Estos fueron comprendidos a partir del método de estudio de casos (Baskarada, 2014; Starman, 2013) con una temática común.

La temática común que compartieron las participantes fue la exposición a acoso laboral en el desarrollo de su trabajo de cuidado en programas psicosociales tercerizados por el Estado chileno.

Cada una de ellas fue considerada en esta investigación como un caso representativo de las consecuencias de la problemática al interior de los programas psicosociales, lo que instó a considerar sus relatos y experiencias como aportes en el reconocimiento y afrontamiento del AMT, toda vez que en los casos se observaron consecuencias en la salud mental y en el ejercicio de sus derechos; así como el acoso laboral alteró el normal desempeño de sus funciones de cuidado e instó a que abandonaran los programas en los cuales se desenvolvían.

\section{Participantes}

La selección de las participantes se realizó de manera intencionada y por conveniencia (Martínez-Salgado, 2012) a través del muestreo por cadena o bola de nieve (Mendieta, 2015) gracias a la difusión de la investigación realizada por el Sindicato Interempresa de trabajadores/as subcontratados/as de SENAME (SINTRASUB-SENAME). Se incluyeron en la muestra las mujeres profesionales mayores de 18 años con más de dos ańos de contrato o vínculo laboral con un programa psicosocial administrado por organismos ejecutores de políticas públicas del Estado chileno. Se excluyeron de la muestra a los varones y a toda persona con vínculo laboral directo con el Estado.

Participaron en este estudio 10 mujeres de edades entre 27 a 45 años. Seis fueron trabajadoras sociales (T.S) y cuatro psicólogas (PS). 
Al momento de experimentar AMT, nueve se desempeñaban en programas psicosociales del Ministerio de Justicia (SENAME), principalmente en el área de prevención del maltrato infantil y una en la línea programática del Ministerio de la Mujer y Equidad de Género (SERNAMEG), del área de acogida a la mujer víctima de violencia. La distribución geográfica fue: uno ubicado en la región de Coquimbo, dos en la región de Valparaíso, dos en la región metropolitana, uno en la región de O’higgins, uno en la región de Maule y tres en la región de Nuble.

\section{Tabla 1}

Variables asociadas a caracteristicas de la muestra y expresión de AMT $(N=10)$

\begin{tabular}{|c|c|c|c|c|c|c|c|c|}
\hline I.D & Edad & $\begin{array}{l}\text { Profe- } \\
\text { sión }\end{array}$ & $\begin{array}{c}\text { Dependencia } \\
\text { Programa }\end{array}$ & $\begin{array}{l}\text { Área de } \\
\text { Trabajo }\end{array}$ & Tipo AMT & $\begin{array}{l}\text { Perpe- } \\
\text { trador }\end{array}$ & $\begin{array}{c}\text { Salida } \\
\text { programa }\end{array}$ & $\begin{array}{c}\text { Solicitud } \\
\text { Ayuda }\end{array}$ \\
\hline E1 & 38 & T.S & MINJU & MI & mixto & mujer & R.V. & $\mathrm{M} / \mathrm{Ps}$ \\
\hline E2 & 42 & T.S & MINMUJEG & V.G & descendente & mujer & Finiquito & No \\
\hline E3 & 32 & PS & $\begin{array}{l}\text { EDUC/ } \\
\text { MINJU }\end{array}$ & M.I & ascendente & mujer & R.V & No \\
\hline E4 & 35 & PS & MINJU & M.I & descendente & hombre & R.V & M/Ps \\
\hline E5 & 28 & PS & MINJU & M.I & descendente & mujer & Finiquito & S \\
\hline E6 & 38 & T.S & MINJU & M.I & descendente & mujer & Finiquito & M/PS \\
\hline E7 & 34 & T.S & MINJU & M.I & descendente & hombre & R.V & M/PS \\
\hline E8 & 27 & PS & MINJU & M.I & descendente & hombre & R.V & M/PS \\
\hline E9 & 27 & T.S & MINJU & M.I & descendente & hombre & R.V & M/PS \\
\hline E10 & 36 & T.S & MINJU & M.I & descendente & hombre & R.V & No \\
\hline \multicolumn{6}{|c|}{ MINJU = Ministerio de Justicia } & \multicolumn{3}{|c|}{ V.G= Violencia de género } \\
\hline \multicolumn{6}{|c|}{ MINMUJEG= Ministerio de la Mujer y Equidad de género } & \multicolumn{3}{|c|}{ R.V = Renuncia voluntaria } \\
\hline \multicolumn{6}{|c|}{ EDUC= Ministerio de Educación } & \multicolumn{3}{|c|}{ M/Ps= Médico-psicólogo/a } \\
\hline \multicolumn{6}{|c|}{ M.I= Maltrato infantil } & \multicolumn{3}{|c|}{$S=$ Sindical } \\
\hline
\end{tabular}

Tal como se puede observar en la tabla 1, la mayoría de las participantes fueron acosadas por un/a superior. Las personas que perpetraron 
las conductas fueron en proporciones similares (50/50) hombres y mujeres. Como no se resolvió adecuadamente la situación y no se prestó atención debidamente al sufrimiento, la totalidad de las participantes abandonó el programa psicosocial, ya sea por renuncia voluntaria o por finiquito. Cabe mencionar que en su mayoría pidieron ayuda especializada y que solo una de ellas se respaldó en las gestiones sindicales para abordar la situación.

\section{Instrumentos}

Se utilizó la técnica de entrevista cualitativa (Gubrium, Holstein, Marvasti, y McKinney, 2012). El guión de la entrevista se diseñó de manera semiestructurada, en torno a tres tópicos. 1) El impacto en la subjetividad al ser víctima de AMT o ¿Cómo impactó en la subjetividad de las víctimas la experiencia de AMT? Con este tópico se intentó suscitar que cada participante realizara comentarios sobre su vivencia particular de AMT; situándose en el desarrollo temporal y social en la que ocurrió; 2) La actuación de sus colegas de trabajo o ¿De qué manera actuó el grupo de pares en el afrontamiento de la experiencia de AMT?, tomando en consideración las estrategias individuales y colectivas de afrontamiento efectuadas por el equipo de trabajo y por último 3) La organización institucional del trabajo de cuidado o ¿Cómo incidieron las condiciones laborales en la experiencia de AMT?, permitiendo que las participantes señalaran los factores facilitadores organizacionales en su vivencia.

\section{Procedimiento}

La construcción de datos y el análisis de resultados se consumaron gracias al uso de un modelo de seis fases, tal como se refiere en la figura 1 . 


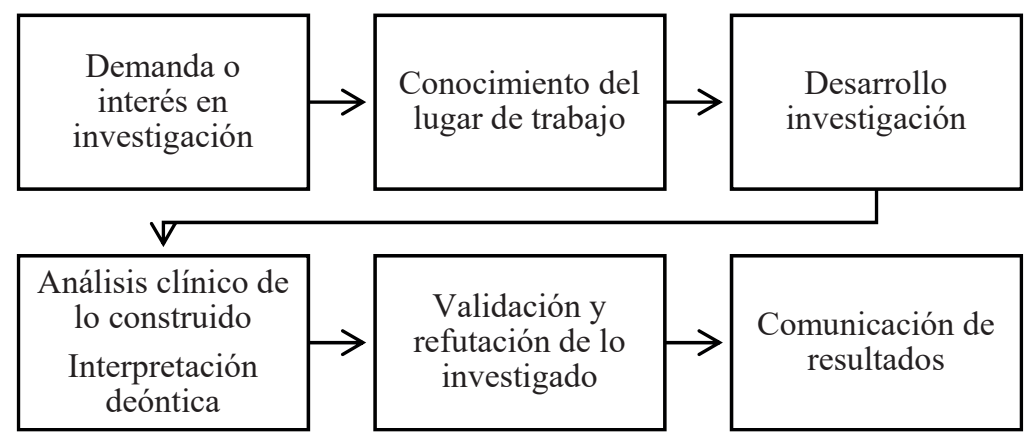

Figura 1. Procedimiento construcción de datos.

La demanda de investigación provino de la directiva de SINTRASUB-SENAME. Los representantes sindicales evidenciaron una queja colectiva, puesto que sus socios/as les hicieron notar que en algunos programas existían situaciones constitutivas de AMT, las cuales afectaban con mayor frecuencia a las mujeres. Por esa razón, el SINTRASUB-SENAME se contactó con los investigadores, quienes acordaron desarrollar la presente investigación, solicitando para ello las gestiones sindicales para contactar a las interesadas. Por resguardo de la identidad de las participantes, se decidió no asistir a sus lugares de trabajo, por lo que se realizó el contacto a través de correo electrónico.

Posteriormente, se efectuó una entrevista con cada participante de una duración de 1 hora y 30 minutos. Estas fueron grabadas y transcritas por el equipo de investigación. Las transcripciones fueron enviadas a las participantes para su revisión, para que validaran y/o refutaran lo escrito. Así, ellas pudieron leerlas haciendo llegar un feedback a los investigadores.

El análisis clínico de los datos construidos se realizó tomando en consideración un mapa temático (ver figura 2). Aquel incorporó tres temas y 13 categorías de análisis. Las categorías emergieron al revisar la literatura relacionada con el AMT. Las transcripciones de las entrevistas se leyeron sucesivamente, se dedujeron las categorías resultantes del AMT en cada caso, se las contrastó con las categorías de análisis y 
se discutieron en relación con los relatos. Para aumentar la rigurosidad, se efectuó triangulación de investigadores.

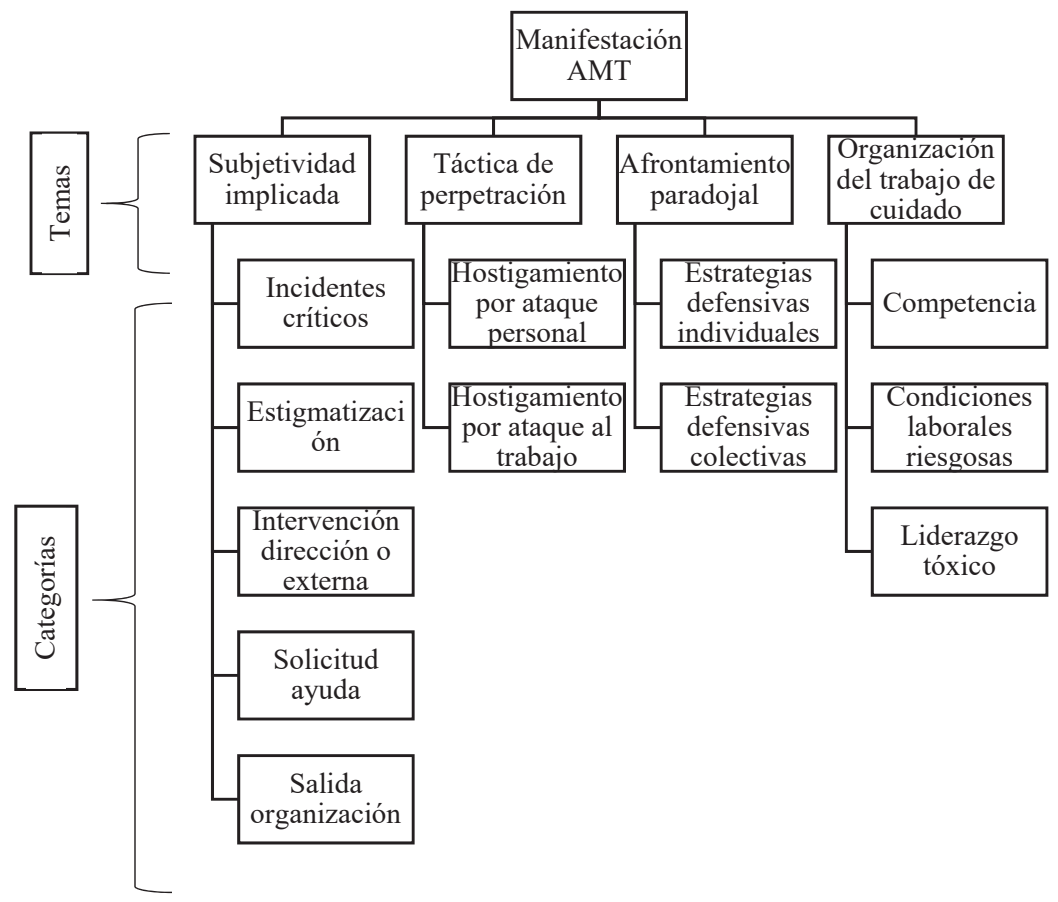

Figura 2. Mapa temático utilizado para el análisis de la manifestación del AMT en los relatos de las participantes.

Se empleó el diseño de análisis del discurso de contenido cualitativo de Bardin (2002). Se hicieron las codificaciones y categorizaciones utilizando el software QDA Miner 5.0. Estas se efectuaron de manera deductiva. En el análisis de lo categorizado se respetaron las sugerencias del análisis temático de Maguire y Delahunt (2017). Se utilizó esta estrategia puesto que permite identificar temas o patrones en los datos que son relevantes para comprender los relatos de las personas. La identificación de temas permite enunciar con mayor claridad algo sobre el 
problema identificado; interpretar el contenido, darle sentido y facilitar su comprensión.

Finalmente, la comunicación preliminar de los resultados se ha realizado en jornadas organizadas por el SINTRASUB-SENAME para la sensibilización en la materia, realizadas en las ciudades de Valparaíso, Quilpué y Santiago.

\section{Consideraciones éticas}

Los resguardos éticos que se cumplieron en esta investigación fueron la confidencialidad de los datos personales de las participantes y la modificación de referencias que facilitaran su identificación. Se entregó información sobre los objetivos del estudio, los riesgos y beneficios a través del consentimiento informado y al inicio de cada entrevista. Cada participante dio su consentimiento por escrito para participar de la investigación. La estructura del consentimiento informado se basó en el prototipo aprobado por el comité de bioética de la Pontificia Universidad Católica de Valparaíso.

\section{Resultados}

A continuación, se presentan los resultados del análisis efectuado según las categorías especificadas en el mapa temático.

Tras la realización del análisis de contenido, las referencias a las condiciones laborales riesgosas en la manifestación del AMT sobresalieron en las narrativas de las participantes. Si aquello se vincula con lo analizado en las categorías de las relaciones de competencia y el ejercicio del liderazgo tóxico, el tema abocado a la forma en que se organiza el trabajo en los programas psicosociales representa un poco más del tercio del total de las menciones realizadas por las participantes como factores elicitadores, mantenedores o explicativos de la exposición a AMT. Esto insta a suponer que en las manifestaciones particulares de AMT, el factor organizacional tuvo una alta incidencia. 
Por su parte, la exposición al AMT implicó que la subjetividad de las trabajadoras se aquejara por un sufrimiento y malestar que no logró ser tramitado y resuelto por la institucionalidad y el grupo de pares. En general, las participantes consideraron que la experiencia fue estigmatizante, caracterizada por una serie de síntomas, por ejemplo, cefaleas, dolores estomacales, aumento de la sensación de angustia, ansiedad y depresión; así como por sus consecuencias sociales, como el aislamiento y abandono realizado por el grupo de pares y el descrédito de lo denunciado. Los incidentes críticos fueron desestabilizantes, toda vez que mantuvieron la hostilidad de los perpetradoras; quienes utilizaron con preferencia el hostigamiento por ataque al trabajo. Una parte importante de las participantes (80\%) refirió haber solicitado apoyo profesional especializado para conseguir licencia médica. Esto, pues la intervención de la dirección de los programas psicosociales fue identificada como una actuación gerencial deficiente y negacionista de la experiencia de AMT que en nada contribuyó a mejorar la situación.

Por último, una parte importante del relato de las participantes se articuló como narración explicativa de las estrategias empleadas para sobrellevar el AMT, destacándose que en la mayoría de los casos se utilizaron estrategias defensivas individuales, puesto que, tal como se mencionó la institucionalidad y el grupo de pares no colaboró a resolver la problemática.

Para facilitar la comprensión de lo descrito anteriormente, en lo que sigue se exponen los resultados del análisis temático efectuado.

\section{La subjetividad implicada en el AMT}

El tema que incorporó estas fases emergió de los relatos con alusión a una subjetividad que se vio afectada por la exposición al AMT, tal como se observa en los casos que siguen.

Los incidentes críticos fueron mencionados por las participantes como eventos que marcaron un punto de inflexión en la relación con la persona perpetradora. A través de estos, las participantes explicaron el inicio de sus experiencias de AMT. 
"Cuando me asignó el cargo [el jefe] me solicitó que me quedara en silencio (...) obviamente los rumores estaban de que yo iba a subir a la jefatura técnica, entonces eso de alguna manera a mis compañeros les causó temor porque al ver a una persona tan cercana y el hecho de que yo no les haya comentado, pese a que a lo que me pidió el director, no les pareció. En su momento yo les logré explicar (...) pero no se logró resolver con una de las delegadas que es la más jovencita y... ahi empezaron mis dificultades" (E1, 22/11/2018)

Estos incidentes críticos también fueron señalados como el comienzo de comportamientos hostiles basados en el disentimiento con las trabajadoras que quisieron ejercer sus derechos laborales, como la libertad de sindicalización y el derecho al goce del fuero postnatal.

"Al final de mayo [2016] me inscribo en el sindicato. Los primeros dias de junio aproximadamente, una semana antes de tener que salir, me indican que mi permiso ya no va, que no corresponde y que me lo van a limitar (...) yo sentía que era por mi afiliación al sindicato, a lo cual mi jefa no pudo negar" (E5, 28/11/2018)

"Yo ingresé a la casa de acogida [2007], yo ingresé con un embarazo de tres meses y que en definitiva fue como el tema que gatilló el maltrato que yo recibi de parte de una directora del SERNAMEG' (E2, $3,25 / 10 / 2018)$

La estigmatización develó la concurrencia de afectos displacenteros que facilitaron la emergencia de síntomas psicosomáticos por el silenciamiento del sufrimiento por temor a perder el empleo. Esto dio cuenta de una pauperización de la salud mental de las participantes, la que se vio afectada por la exposición al hostigamiento.

"Yo empecé a tener sintomas. Para empezar, no quería ir a trabajar, le contaba a mi mamá (...) empezaba a sentir triste, me sentía nerviosa, le tenía miedo. Ahi le tenía mucho miedo, ella [perpetradora] ejercía mucho poder, mucho poder, entonces podia despedirme en cualquier momento yo tenia miedo de que me despidieran, entonces fui aguantando" (E3, 2, 26/11/2018) 
Por último, la estigmatización tendió a facilitar el aislamiento de las víctimas y las incitó a soportar individualmente el malestar. En un caso se observó la nocividad de soportar la hostilidad característica del AMT.

[el acoso] "fue como todo el rato resistir, resistir, resistir entonces todo este tiempo, el trastorno adaptativo tiene que ver con eso, como con el cansancio crónico, de la resistencia que es muy natural en mí, pero también todos nos cansamos no más, entonces eso (...) y claro como que yo me siento a veces como un poco como Juana de Arco" (E4, 9, 27/11/2018)

Al ser estigmatizadas, algunas de las participantes señalaron que recurrieron a la intervención de la dirección del programa psicosocial o de agentes externos cuando la situación de AMT se les hizo insoportable. Sin embargo, en sus relatos clarificaron que la intervención realizada estuvo orientada a cuestionar lo denunciado

"Comuniqué la situación a mi empleador, pero no tomó ninguna medida para resguardar, al contrario, creo que eh... me vi más cuestionada yo...porque pese a que mis compañeros, yo entiendo, ellos, todos presenciaban la situación" (E1, 6, 22/11/2018)

"Fui a la Inspección del Trabajo, atendió un abogado, le tuve que dar todo el discurso de lo que sucedía en mi trabajo y jél me escuchaba como abogado poh! Él trataba de discernir entre las situaciones que él veía como acoso o no, si habia alguna situación de derechos fundamentales o no, entonces jtambién fue difícil para mi esa cuestión poh?' (E8, 12, $26 / 10 / 2018)$

Mientras que la intervención institucional resultó deficiente, las participantes requirieron de apoyo profesional especializado con la finalidad de abordar su sintomatología. Por la severidad, los especialistas les prescribieron licencia médica.

"Empecé a buscar ayuda psicológica porque esta inseguridad como de Hoy no me siento bien, jah! pero ya, igual siéntete bien-como que eso me preocupó, como de no validar lo que yo estaba sintiendo (...) Entonces, 
como que empecé a buscar ayuda y como que ahi antes de irme empecé una terapia que sostengo hasta hoy en día" (E9, 7, 09/11/2019)

"Yo voy a mi control de Ginecólogo y me aceleran me anticipan mi prenatal porque el médico no podía creer que yo trabajaba en esas condiciones con mujeres víctimas de violencia grave, entonces me adelanta el prenatal' (E2, 10, 23/11/2018)

\section{Las tácticas de perpetración}

Las acciones de hostigamiento fueron dirigidas por perpetradores de ambos sexos. Estas se desarrollaron siguiendo la tipología de AMT referida por Hirigoyen (2013). Las tácticas de hostigamiento fueron por ataque personal y por ataque al trabajo.

En cuanto al hostigamiento por ataque personal, se caracterizó por la realización de amenazas, actos o comentarios de menosprecio para atacar la valía y la capacidad de esfuerzo personal.

"Entonces una vez ella [acosadora] me dijo así poh, que cómo se me ocurría quedar embarazada con el sueldo que yo tenía que andaba alegando y que no me iba a alcanzar para otro hijo y eso me lo dijo en reunión con toda la gente de mi programa" (E10, 6, 15/11/2018)

"Estábamos capacitando a las educadoras de trato directo (...) cuando llega la directora [del programa] y nos dice que nosotras somos unas flojas, en especial yo porque no teniamos mujeres en la casa, no teníamos victimas [de violencia]" (E2, 8, 25/10/2018)

Los recursos que utilizaron los/as perpetradores fueron la seducción y la desestabilización psicológica experimentadas por las víctimas como confusión y desagrado. En algunos casos, recurrieron a formas de violencia por motivo de género, como el acoso sexual y por motivo de maternidad, tal como se aprecia en los siguientes extractos.

"Un embarazo de siete meses que yo oculté, ya, porque tenía mucho miedo de que la directora me despidiera (...) Un día no lo pude esconder más poh, todo el equipo sabia que yo estaba embarazada, pero yo no lo pude esconder porque la guata me creció enormemente y se aparece 
con dos colaboradoras de ella (...) me dice: ¿estás embarazada? y me queda mirando así con una cara de odio" (E2, 5, 23/11/2018)

"Si él [perpetrador] me decía algo, pero nunca me... ponte tú, era súper fresco igual, le tiraba comentarios a todas las cabras les decía algo, jay! no sé, ya no me acuerdo como les decía. Mi sirenita ponte tú, pero a la vez era como súper drástico (...) era el gallo que la tiraba eso pero después les exigía otra cosa" (E4, 10, 27/11/2018)

Este caso representa la gravedad del hostigamiento, luego que el perpetrador cercenara los vínculos sociales de la víctima y la responsabilizara de las fallas de la institución para supervigilar el cumplimiento de la licencia médica; asumió perversamente la función de vigilancia y se entrometió en su vida privada.

Las tácticas de hostigamiento por ataque al trabajo incluyeron el aumento de la carga de trabajo y de la responsabilidad en la concreción de las tareas, además de la imposición de metas o instigación a su cumplimiento.

"El nivel de estrés que viven funcionarios de la red SENAME, en general o cualquier profesional que trabaje con violencia tan alto que te deja susceptible emocionalmente a situaciones de maltrato, como situaciones de autoritarismo y que ese mismo estrés hace que las autoridades también te sobre-exijan (...) yo creo que tiene mucho que ver con el poder manifestar que se cumplieron ciertas metas o que la eficacia está, del trabajo, como muy fijado en el producto, muy centrado en el cumplimiento de tareas" (E5, 28/11/2018)

En otras experiencias de AMT, el ataque al trabajo se realizó para inducirlas al error $\mathrm{u}$ obstruir el correcto ejercicio de sus funciones y denegar derechos laborales. En un caso, aquello involucró la realización de un procedimiento negligente.

"Una joven que fue víctima de una violación, obviamente ese era un caso que procedia que yo estuviera como jefe técnico y esta niña [perpetradora] no me informó de nada, hicieron todo a su manera, cosa que no correspondia y obviamente como no manejaban los conocimientos pertinentes, quedó la embarrá con el caso..." (E1, 3, 22/11/2018) 
Al exigirles más exhaustividad en las tareas, aumentaron los errores técnicos, los cuales eran señalados insistentemente para cuestionar la idoneidad profesional en el desempeño de las funciones.

"Era un desmedro porque ella me hacia hacer cosas que no me correspondian dentro de mi cargo y yo hacia más de lo correspondía y aun asi ella encontraba que estaba mal, me acuerdo una vez que [directora] me evaluó con un dos cinco en una escala del uno al siete" (E10, 3, $15 / 11 / 2018)$

Finalmente, sobre la utilización de las acciones de ataque al trabajo, algunas participantes comentaron que las prácticas de hostigamiento fueron dirigidas hacia ellas para justificar la denegación de derechos

"Me manifestaron que yo no podía tomarme más días administrativos, yo la verdad es que en ese momento lo entendí bueno, tengo un contrato a plazo fijo quizás por eso, como tratando de evitar, racionalmente sentirme acosada laboralmente" (E5, 6, 28/11/2018)

\section{Afrontamiento paradojal del AMT}

En el afrontamiento de las experiencias de AMT, las participantes mencionaron que utilizaron diferentes estrategias defensivas para proseguir con sus funciones normalmente. Esta normalidad en el sufrimiento (Dejours, 2009a) se articuló gracias al uso de estrategias defensivas individuales y colectivas que sirvieron para tolerar las prácticas de hostigamiento. Algunas participantes señalaron que la exposición al AMT se soportó mejor cuando fueron capaces de enmudecer o silenciar su malestar; mientras que otras, a pesar de su oposición activa al inicio, terminaron por ceder y salir del programa psicosocial.

Las estrategias defensivas individuales utilizadas por las participantes fueron referidas como negación del malestar, así como reanimación de su capacidad para sobrellevar la experiencia de AMT.

"Mira, a veces me silenciaba, a veces prefería como invisibilizarme frente al resto, pero la verdad es que no me era muy efectivo porque igual sentía yo que buscaba constantemente que yo diera opiniones para poder tener instancias como de confrontación" (E5, 4, 28/11/2018) 
"Yo siempre me mantuve trabajando, como que ahi no estaba el problema, como que sentía que tenía una responsabilidad muy grande encima, como habia tanta pega atrasá, yo de verdad quería como que las cosas se solucionaran ¿cachai?, más que por el proyecto o la fundación era por los chiquillos" (E9, 5, 09/11/2018)

En algunas alusiones a los afectos displacenteros que debían tramitar con esas estrategias defensivas, las participantes seńalaron que tenían relación principalmente con el ejercicio del cuidado. En ciertos casos se observó que las direcciones de los programas les solicitaron a las trabajadoras ejecutar acciones contrarias a la ética profesional. Estas solicitudes atentaron contra el sentimiento de contribución de cuidado a las víctimas

"Cuando ella fue insistente en ese sentido nosotras egresamos a algunas mujeres, pero la teníamos de vuelta a las tres o cuatro semanas y con un nivel de agresión mucho mayor en ellas, entonces yo en ese sentido me siento bastante responsable por lo que a esas mujeres les ocurrio" (E2, $5,23 / 11 / 2018)$

Las estrategias defensivas individuales se vieron favorecidas cuando operaron en relación con las estrategias defensivas colectivas. La inhibición de la acción colectiva para enfrentar el malestar común con la organización, la solidaridad cínica del grupo con las víctimas, el trabajo sucio para tutelar las normas institucionales y el desprecio de las trabajadoras benefició la banalización colectiva del sufrimiento.

La inhibición de la acción colectiva se vio reflejada en que el grupo de pares desistió en intervenir a favor de la resolución del problema. No tomaron ninguna medida que disipara al AMT

"Cuando yo inicié con mi licencia, todos sabian que yo no iba a volver más y me mandaron whatsapp así como: que lo siento mucho, que esto no debió de haber sido para ti, no correspondía lo que viviste, todos sabiamos que lo estabas pasando mal, igual todos reconocieron lo que yo habia vivido, pero obviamente yo entiendo, que necesitan trabajar, todos necesitamos trabajar y yo tampoco iba a esperar que se la jugaran por mì'(E1, 14, 22/11/2018) 
En algunos casos, cuando el grupo de pares se compadeció por el sufrimiento de las trabajadoras, realizaron algunas actividades de apoyo emocional. Sin embargo, ese respaldo solidario fue comprendido por las participantes como un cinismo grupal, pues en rigor no pudo detener las conductas de hostigamiento.

"Yo creo que a pesar de que ellos no me ayudaban de forma explícita, eran dos personas las que estaban siempre conmigo, que me decian: no, tienes que estar tranquila, hagamos esto, porque tampoco podian hacer algo, ellas también tenian temor de que les hicieran algo. Entonces yo creo que también me sostuve por ellas porque también alargaron esta agonía y yo también me dejê" (E3, 6, 26/11/2018)

La ausencia de un afrontamiento colectivo que detuviera el AMT contribuyó a que el trabajo sucio se mantuviera. El sostenimiento de una relación de poder basada en el desprecio viril o la muliebridad defensiva fueron expresiones de la banalización colectiva del AMT.

"Los que son más cercanos si se daban cuenta de la situación [de AMT], criticaban a la jefatura, si me entregaban palabras de ánimo, pero en los momentos de reuniones donde estaba el equipo completo y se vivian situaciones como de injusticia hacia mí, o me indicaban que no me podia tomar los dias de permiso administrativo, si bien todos silenciosamente dijeron: o que mal eso, no corresponde, nadie alzó la voz para apoyarme, como explícitamente" (E5, 9, 28/11/2018)

\section{Organización del trabajo de cuidado en las experiencias de AMT}

Las experiencias de AMT también fueron comentadas como entrecruzamiento de distintos factores de riesgo presentes en los programas. Aquellos fueron aludidos como facilitadores en la emergencia y mantención del AMT.

"Yo creo que eso se asocia mucho a las condiciones laborales que ofrecen este tipo de programas, yo creo que tiene situaciones tan complejas que se requieren de profesionales mejor preparados, yo creo que eso, de alguna manera no todos lo pueden abordar de manera cómo...éticamente, 
profesionalmente, como minuciosamente hablando desde la ética profesional' (E1, 13, 22/11/2018)

Las relaciones de competencias en el trabajo fueron referidas como elicitadoras de la violencia al interior del grupo de pares, con un fuerte basamento en actitudes patriarcales

"Habia una cierta politica institucional diría yo que fomentaba bastante esta lógica de individualismo de un poco como salvarse cada uno, como de preocuparse mucho cada uno de la pega (...) en el cotidiano se convertía en una especie de combate o de competencia constante con tus compañeros, estaba muy presente esa dinámica o esa lógica de trabajo" (E6, 2, 21/11/2018)

"A parte de ser una visión súper patriarcal [del programa], asi terrible y además tienen tan poco conocimiento, son tan poco... hay poca reflexión, hay muy poca reflexión, hay muy poca, bueno la critica no existe, hay mucho de justificar los actos, no sé poh, si puedo cagar al del lado, lo hago y me puedo salvar yo, entonces hay una cosa egoísmo, individualismo" (E4, 17, 27/11/2018)

En otros casos, las alusiones a condiciones laborales riesgosas fueron importantes para explicar sus experiencias de AMT. Se destacaron referencias a la alta demanda emocional para el trabajo con población victimizada, la precarización laboral, la orientación al cumplimiento de metas, actividades de cuidados de equipo poco efectivas o atingentes a sus necesidades, falta de claridad en las funciones y tareas, falta de capacitación y preparación para abordar el trabajo.

"La cultura del rendimiento que lo único que importa es que da lo mismo cómo se siente el otro, trabajas de forma mecánica, trabajas con el piloto automático todo el tiempo, ya trabajas, trabajas, trabajas, que es un número esta cuestión, entonces eso te hace ser mecánico y te hace ser violento, yo por lo menos lo veo asi y es lo que dicen mis colegas, gran número de casos que hay en SENAME pocas capacitaciones y un montón de cosas" (E3, 13, 26/11,2018) 
El ejercicio de la autoridad controladora y las supervisiones abusivas en los programas tuvieron un impacto importante en la manifestación de AMT. El liderazgo basado en la asimetría de poder y orientación al cumplimiento de indicadores se personificaron en los/as perpetradores para hostigar a las participantes.

\section{Discusión}

Los resultados expuestos dieron cuenta de la manifestación del AMT en mujeres que realizaban trabajo de cuidado con víctimas de violencia en programas psicosociales del SENAME y SERNAMEG en Chile.

En cuanto a la subjetividad implicada en las experiencias, los resultados sobre la exposición a incidentes críticos son concordantes con una investigación realizada por López y Seco (2016) en el contexto organizacional español. En algunos casos analizados en este estudio, las conductas de hostigamiento comenzaron por conflictos de derechos, tanto en lo referido a la participación sindical como en el ejercicio del descanso maternal.

Por su parte, los hallazgos en torno a la estigmatización por AMT se relacionan con los resultados de una investigación realizada por da Silva y Saldanha (2017) con enfermeras de servicios de atención en Portugal. En los resultados de este estudio, las mujeres relataron haber experimentado diversos síntomas psicosomáticos. Ese malestar incidió en su desvalorización personal y en el aislamiento del grupo de pares, lo que afectó su salud mental. Estas incidencias se manifestaron en dolores agudos, incrementándose la sensación de angustia y el sufrimiento. Para detener la cronificación de ellos, las participantes solicitaron apoyo externo de médicos, quienes les otorgaron licencia médica y medicamentos.

Sobre la intervención de la dirección o externa, los resultados son concordantes con la investigación realizada por Tinuke (2013) en cuatro sectores productivos de Nigeria. En los casos analizados en este 
estudio, las direcciones de programas y agentes externos tendieron a invisibilizar, dificultar o negar la situación.

Al proseguir las conductas de hostigamiento sin control por parte de las direcciones, las trabajadoras recurrieron al apoyo especializado para conseguir licencias médicas o consejos legales. Este resultado se asocia con los comentarios de una revisión realizada por Ramírez y Mayorga (2017) sobre riesgos laborales psicosociales.

El uso de las tácticas de perpetración supone que primaron los ataques al trabajo por sobre los ataques personales. Estos resultados son afines con un estudio realizado por Domínguez et al. (2012) con trabajadores de atención en salud en España.

En este estudio, la utilización de las tácticas de ataque al trabajo tuvo como consecuencia la sensación de agobio laboral, incumplimiento de tareas, aumento de equívocos y faltas profesionales. Los hostigamientos por ataque personal se ejercieron mediante amenazas, humillaciones, menosprecio y denigración por motivos de género, como la seducción perversa y la desvalorización de las cualidades para el cuidado. En un caso se recurrió al acoso sexual; en otros dos a discriminación por motivos de maternidad y orientación política.

Los recursos defensivos del AMT más destacadas fueron los individuales; como la negación y enmudecimiento del sufrimiento, realización de actividades de compensación y finalmente la abnegación para el cuidado de otros mientras se sobrellevaba el AMT. Los resultados se relacionan con los de Palma, Ahumada, y Ansoleaga (2018) sobre formas de afrontamiento pasivo individual de la violencia laboral en trabajadores chilenos.

Respecto al uso de estrategias defensivas colectivas, se observó inhibición de la acción colectiva para afrontar el AMT, solidaridad basada en el cinismo y banalización del sufrimiento. Esto es concordante con los resultados de la investigación ejecutada por Campos, David y Souza (2014) sobre experiencias de sufrimiento y placer de enfermeras del sector de cuidados intensivos, donde las estrategias hicieron tolerable el sufrimiento silenciándolo colectivamente. 
Finalmente, sobre las alusiones al rol de la organización del trabajo de cuidados en las experiencias de AMT, las participantes resaltaron las relaciones interpersonales basadas en la competencia y en actitudes machistas; así como en condiciones laborales caracterizadas por riesgos psicosociales, como la precariedad laboral, el desgaste emocional o burnout, presión por cumplimiento de metas, el ejercicio de liderazgos o supervisiones autoritarias y tóxicas. Esta serie de factores son concordantes con la revisión sistemática sobre factores facilitadores de la violencia laboral realizada por Toro y Gómez (2016); con el estudio sobre burnout en profesionales de programas psicosociales chilenos realizado por Bilbao et at. (2018) y en una tesis doctoral realizada por Pavez (2018) sobre precariedad laboral en esos mismos programas.

\section{Conclusiones}

Los resultados presentados y discutidos en función de la evidencia científica permiten compartir conclusiones sobre las determinaciones y consecuencias de la manifestación de AMT en mujeres chilenas que realizaban trabajo de cuidado con víctimas de violencia.

Las determinaciones fueron las condiciones laborales en los programas psicosociales, catalogadas como facilitadoras y mantenedoras de la violencia. Lo discutido insta a suponer que la organización del trabajo de cuidado en programas psicosociales, en la medida que propicia la precariedad laboral, el ejercicio de liderazgos destructivos y relaciones de competencia puede actuar como escenario de desarrollo y cronificación de AMT.

Las consecuencias del AMT se visualizaron en una afección en la salud mental y en el ejercicio de derechos de las mujeres, lo que impidió que desarrollaran adecuadamente sus funciones. Esto se observa como un resultado paradojal, puesto que la política pública nacional busca brindar apoyo psicosocial especializado a víctimas de violencia. Sin embargo, en estos programas el AMT actuaría como promotor de violencia contra las mujeres trabajadoras y contribuiría a la alta rota- 
ción laboral en el rubro (Pavez et at, 2016), dado su acicate para que las víctimas salgan de la organización.

En relación a las limitaciones de este estudio, se pueden señalar que los resultados no necesariamente se pueden generalizar al conjunto de trabajadores del rubro, porque en esta investigación se priorizó comprender la manifestación de AMT en las trabajadoras, lo que mantiene en el desconocimiento la problemática en hombres $\mathrm{u}$ otras identidades de género. Por lo anterior, se sugiere continuar profundizando el estudio del AMT en el área. Cobra relevancia efectuar una pesquisa sobre la magnitud del problema a nivel nacional.

\section{Referencias}

Albanaes, P., Roque, J., Gasperin, P. y da Rosa, S. (2017) Intervenção em grupo de apoio psicológico a trabalhadores vítimas de assédio moral. Revista de Psicología (Perú), 35(1) 61-96. http://dx.doi. org/10.18800/psico.201701.003

Appelbaum, S., Semerjian, G. y Mohan, K. (2012), Workplace bullying: consequences, causes and controls (part one), Industrial and Commercial Training, 44(4), 203-210 http://dx.doi. org/10.1108/00197851211231478

Appelbaum, S., Semerjian, G. y Mohan, K. (2012). Workplace bullying: consequences, causes and controls (part two), Industrial and Commercial Training, 44(6) 337-344 http://dx.doi. org/10.1108/00197851211254770.

Bardin, L. (2002) El análisis de contenido. Akal.

Baskarada, S. (2014). Qualitative Case Study Guidelines. Qualitative Report, 19(40), 1-25. https://doi.org/10.7748/ nr2013.05.20.5.28.e327

Bassi, J. (2014). Cuali/Cuanti: La distinción paleozoica. Forum Qualitative Sozialforschung, 15(2). Recuperado de http://nbn-resolving. de/urn:nbn:de:0114-fqs140279. 
Bilbao, M., Martínez, G., Pavez, J., Morales, K. (2018). Burnout en trabajadores de ONGs que ejecutan política social en Chile. Psicoperspectivas, 17(3), 1-12. https://doi.org/10.5027/ psicoperspectivas-Vol17-Issue3-fulltext-1454

Branch, S., Ramsay, S., y Barker, M. (2013). Workplace bullying, mobbing and general harassment: A review. International Journal of Management Reviews, 15(3), 280.299. https://doi. org/10.1111/j.1468-2370.2012.00339.x

Campo, V. R., y Klijn, T. P. (2018). Verbal abuse and mobbing in pre-hospital care services in Chile. Revista Latino-Americana de Enfermagem, 25(0), 1-8 https://doi. org/10.1590/1518-8345.2073.2956

Campos, J., David, H., y Souza, N. (2014). Pleasure and suffering: assessment of intensivist nurses in the perspective of work psychodynamics. Escola Anna Nery - Revista de Enfermagem, 18(1), 90-95. https://doi.org/10.5935/1414-8145.20140013

Castellón, A. M. (2012). Hostigamiento laboral: amenaza permanente para enfermería. Enfermería Global, 11(28), 120-136. Recuperado de http://scielo.isciii.es/scielo.php?script=sci_pdf\&pid=S1 695-61412012000400008\&lng=es\&nrm=iso\&tlng=es

Chappell, D. y Di Martino, V. (2006). Violence at work. International Labour Organization.

da Silva, A. y Saldanha, A. F. (2016). Mobbing and Its Impact on Interpersonal relationships at the Workplace. Journal of Interpersonal Violence, 34(13), 2797-2812. https://doi. org/10.1177/0886260516662850

Dejours, C. (2006) La banalización de la injusticia social. Topía.

Dejours, C. (2009a) Trabajo y sufrimiento. Cuando la injusticia se hace banal. Modus Laborandi

Dejours, C. (2009b) El desgaste mental en el trabajo. Modus Laborandi. Dejours, C. (2014). Work and Self-Development. Critical Horizons, 15(2), 115-130. https://doi.org/10.1179/1440991 $714 \mathrm{Z} .00000000027$ 
Dejours, C. y Gernet, I. (2012). Psicopatología del trabajo. Miño y Dávila.

Díaz, X., Mauro, A., Ansoleaga, E., Toro (2017). Violencia de género en el trabajo en Chile. Un campo de estudio ignorado. Ciencia \& Trabajo, 19(58), 42-48. https://doi.org/10.4067/ S0718-24492017000100042

Domínguez, J., Padilla, I., Domínguez, J., Martínez, M., Ortega, G., García, M. y Moreno, A. (2012). Acoso laboral en trabajadores de atención a la salud de Ceuta. Medicina y Seguridad Del Trabajo, 58(227), 117-127. https://doi.org/10.4321/ S0465-546X2012000200005

dos Santos, T y Ducatti, I. (2014). Dividir para reinar: las relaciones de género en el acoso moral en el trabajo. Salud de los Trabajadores, 22(2), 141-152. Recuperado de http://www.scielo.org.ve/scielo. php?script=sci_arttext\&pid=S1315-01382014000200005

Eurofound. (2015). Violence and harassment in European workplaces: Extent, impacts and policies. Eurofond.

Fardella, C., Sisto, V., Morales, K., Rivera, G. y Soto, R. (2016). Identidades laborales y ética del trabajo público en tiempos de rendición de cuentas. Psykhe, 25(2), 1-11. https://doi. org/10.7764/psykhe.25.2.789

Fernández, M., López, T., Espinoza, D., Velasco, M., Osorio, L. y Radillo, B. (2012). Autopercepción de presuntos perpetradores de acoso psicológico en el trabajo. Un acercamiento fenomenológico. Salud Uninorte, 28(1), 27-35. Recuperado de http://rcientificas.uninorte.edu.co/index.php/salud/article/ viewArticle/3203/3696

Gubrium, J., Holstein, J., Marvasti, A. y McKinney, K. (2012). Interview Research. The Complexity of the craft. Sage Publications.

Hernandez, T. (2014). Empresas libres de violencia: prevención, detección, atención y sanción de la violencia basada en género y el acoso sexual y laboral. PNUD.

Hirigoyen, M.F (2013) El acoso moral en el trabajo. Paidós. 
Hirigoyen, M. F. (2016). Le harcèlement moral, un symptôme de la société moderne. Annales Medico-Psychologiques, 174(7), 575-579. https://doi.org/10.1016/j.amp.2016.05.004

Krasikova, D. V., Green, S. G., y LeBreton, J. M. (2013). Destructive Leadership: A Theoretical Review, Integration, and Future Research Agenda. Journal of Management, 39(5), 1308-1338. https://doi.org/10.1177/0149206312471388

Leymann, H. (1990). Mobbing and Psychological terror at workplaces. Violence and Victims, 5(2), 119-126. Recuperado de https:// www.ncbi.nlm.nih.gov/pubmed/2278952

Leymann, H. (1996). The Content and Development of Mobbing at Work. European Journal of Work and Organizational Pshychology, 5(2), 165-188. https://doi.org/10.1080/13594329608414853

López, C. y Seco, E. (2016). Tipologia de Mobbing - una mirada desde la responsabilidad de la empresa. Sociologias, 18(43), 364-401. http://dx.doi.org/10.1590/15174522-018004321

Luiz, E., Barlem, D., Lunardi, V. L., Lerch Lunardi, G., Tomaschewski, J.y Silva, R. (2013). Sufrimiento moral en el cotidiano de la enfermería: huellas ocultas de poder y resistencia. Revista LatinoAmericana de Enfermagem, 21(1), 1-8. Recuperado de http:// www.scielo.br/pdf/rlae/v21n1/es_v21n1a02.pdf

Madrigal, E. y Calderón, J. (2015). Revisión bibliográfica conceptualización médico legal del acoso laboral, Medicina Legal de Costa Rica, 32(2), 1-10. Recuperado de http://www.scielo.sa.cr/pdf/ $\mathrm{mlcr} / \mathrm{v} 32 \mathrm{n} 2 / \operatorname{art} 08 \mathrm{v} 32 \mathrm{n} 2 . \mathrm{pdf}$

Maguiere, M. y Delahunt, B. (2017). Doing a thematic analysis: A practical, step-by-step guide for learning and teaching scholars, AISHE-J, 9(3), 1-14. Recuperado de http://ojs.aishe.org/index. $\mathrm{php} /$ aishe-j/article/view/335

Martínez, C. (2012). El muestreo en investigación cualitativa. Principios básicos y algunas controversias. Ciência \& Saúde Coletiva, 17(3), 613-619. https://doi.org/10.1590/ S1413-81232012000300006 
Mendieta, G. (2015). Informantes y muestreo en investigación cualitativa. Investigaciones Andina, 17(30), 1148-1150. Recuperado http://www.redalyc.org/pdf/2390/239035878001.pdf

Molina, N y Jung, J. (2015). Estudio del acoso psicológico laboral descendente a mujeres y su Incidencia en el Ausentismo empresarial. Investigación Psicológica, 14, 19-42. Recuperado de http://www.scielo.org.bo/scielo.php?script=sci_arttext\&p $\mathrm{id}=$ S2223-30322015000200004

Molinier, P. (2010). Souffrance, défenses, reconnaissance. Le point de vue du travail. Nouvelle Revue de Psychosociologie, 10(2), 99. https://doi.org/10.3917/nrp.010.0099

Molinier, P y Legarreta, M. (2016). Subjetividad y materialidad del cuidado: ética, trabajo y proyecto político. Papeles Del CEIC, (1), 1-14. https://doi.org/10.2166/wst.2011.453

Organización Internacional del Trabajo (2018). Acabar con la violencia y el acoso en el mundo del trabajo. ILO.

Organización Mundial de la Salud (2015) Plan de acción sobre la salud de los trabajadores 2015-2025. WHO.

Palma, A., Ahumada, M., y Ansoleaga, E. (2018). ¿Cómo afrontan la violencia laboral los trabajadores / as chilenos / as?. Psicoperspectivas, 17(3), 1-13. https://doi.org/http://dx.doi.org/10.5027/ psicoperspectivas/vol17-issue3-fulltext-1288

Pavez, J., Carrasco, C., Peña, M., Bilbao, M., Oriol, X., Rubio, A., Torres, J. (2016). El comedor: las investigaciones llegan a la mesa. Sujeto/a trabajador/a en la política pública de intervención psicosocial de infancia en Chile: un análisis crítico del discurso. Revista de Estudios Cotidianos, 1(4), 23-45. Recuperado de https:/dialnet.unirioja.es/descarga/articulo/5568032.pdf

Pavez, J. (2018). Significados y prácticas de la precariedad en trabajadores y trabajadoras de un programa social de infancia en la región de Valparaiso (Tesis doctoral). Valparaíso: Pontificia Universidad Católica de Valparaíso. 
Quintana, C (2005) El síndrome de burnout en operadores y equipos de trabajo en maltrato infantil grave. Psykhe, 14(1), 55-68. http:// dx.doi.org/10.4067/S0718-22282005000100005

Ramírez, A. y Mayorga, D. (2017). Riesgos laborales psicosociales. Perspectiva organizacional, jurídica y social. Revista Prolegómenos, 20(40), 159-172. https://doi.org/10.18359/prole.3047 Forma

Ros, C. (2013). Violencia y acoso laboral en trabajadores del sector servicios. V Congreso Internacional de Investigación y Práctica Profesional en Psicología. Facultad de Psicología. Buenos Aires: Universidad de Buenos Aires. Recuperado de https://www.aacademica.org/000-054/388\%0AActa

Santana, I. y Farkas, Ch. (2007). Estrategias de autocuidado en equipos profesionales que trabajan en maltrato infantil. Psykhe, 16(1), 77-89. http://dx.doi.org/10.4067/ S0718-222820070001000249

Sepúlveda, A. C., Mota, A. R., Fajardo, G. E., y Reyes, L. I. (2017). Workplace bullying during specialty training in a pediatric hospital in Mexico: a little-noticed phenomenon. Revista Médica del Instituto Mexicano del Seguro Social, 55(1), 92-101. Recuperado de http://www.ncbi.nlm.nih.gov/pubmed/28212481

Sisto, V. y Fardella, C. (2008). Narrándose en la flexibilidad. Un análisis narrativo discursivo de la identidad en tiempos de flexibilidad laboral. Revista de Psicología, 16(2), 56-80. http://dx.doi.org/10 .5354/0719-0581.2011.17137

Starman, A. B. (2013). The case study as a type of qual research, Jounal of Contemporary Educational Studies, 1, 28-43. Recuperado de http://www.sodobna-pedagogika.net/wp-content/ uploads/2013/03/Starman1.pdf

Tepper, B., Simon, L y Park, H. M. (2017). Abusive Supervision. Annual Review of Organizational Psychology and Organizational Behavior, 4, 123-152. https://doi.org/10.1146/ annurev-orgpsych-041015-062539 
Tinuke. M., F. (2013). Managing Workplace Bullying. Journal of Human Resource Management, 1(3), 39-47. https://doi.org/10.11648/j. jhrm.20130103.11

Toro, J. P. y Gómez, C. (2016). Factores facilitadores de la violencia laboral: una revisión de la evidencia científica en América Latina. Ciencia \& Trabajo, 18(56), 110-116. http://dx.doi.org/10.4067/ S0718-24492016000200006

Enviado: 2019-06-19

Revisado: 2021-07-30

Aceptado: 2021-11-19 